\title{
Experiences of an Ethnographer in Two Ghanaian Hospitals
}

\author{
Jonathan Mensah Dapaah \\ Department of Sociology and Social Work \\ Kwame Nkrumah University of Science and Technology \\ Ghana
}

\begin{abstract}
In Ghana and many sub-Saharan African countries, ethnographies of medical setting are relatively uncommon. This might be due to the general view that to study the area of biomedicine by investigating hospital, health centre or clinic is a new territory for medical anthropologists. Besides, perhaps, anthropologists rarely made biomedicine itself a subject for analyses and accepted that it is supposedly grounded in science; it is not a subject of anthropological or sociological inquiry. Therefore, anthropologists over the years devoted much of their time into studying rituals and beliefs of exotic cultures, indigenous therapists, lay perceptions of health and illness. However, there is a growing interest in recent years in studying biomedicine as a cultural or social system. In this regard, this article seeks to illustrate the rigorous processes/procedures social scientists need to go through to enter the hospital and the peculiar strategies they have to adopt in order to collect data in the hospital setting. Further, it tries to show how actors in the facility (which includes biomedical professionals, patients and their relatives) generally perceive social scientists who conduct social science research and particularly ethnography in the hospital setting. The article thus sheds light on the experiences of an ethnographer in the hospital and how he continuously negotiated his presence to collect data in a setting which is often considered the domain of biomedical professionals. The article concludes that the anthropological method is not common in the hospital setting and therefore the need for more hospital ethnography to make it popular among health workers and also demonstrate its important contribution to quality health care delivery.
\end{abstract}

Keywords: HIV/AIDS, counseling, testing, treatment, ethnography

\subsection{Introduction}

There is a general paucity of literature on ethnographic studies in the hospital or health care setting in Ghana and many sub-Saharan African countries. This might be due to the general view that health field research is generally quantitative and based on biomedical traditions and experimental methods. Therefore, in this field, qualitative research is criticised for being subjective to researcher bias and for lack of reproducibility and general is ability (Patton 2002).To study the area of biomedicine by investigating hospital, health centre or clinic is a new territory for social scientists especially medical anthropologists (Van der Geest \& Finkler 2004).

Besides, perhaps, anthropologists rarely made biomedicine itself a subject for analyses and accepted that it is supposedly grounded in science; it is not a subject of anthropological or sociological inquiry. As a result, anthropologists preferred to study cultures and groups in their own countries of origin and foreign settings and told stories of their experience in the past (Holloway 1997). There is also the view by Goodson and Vasser (2011) that qualitative research which is akin to ethnography is often overlooked as an option when considering the methodological approach to research in the health care setting. This, according to the authors is especially true in academic domains such as medicine where evidence-based practice has emerged as a popular treatment philosophy based on largely quantitative research tradition.

Perhaps more importantly, the observation by Van der Geest and Finkler (2004) that many anthropologists have come to realize that in a hospital, participant observation which is a key data collection method in ethnographic studies intertie sense of the termisan oxymoron due to the peculiar nature of the hospital setting which has mainly health professionals most of who are largely not use to qualitative research methods. Therefore, anthropologists over the years devoted much of their time into studying rituals and beliefs of exotic cultures, indigenous therapists, lay perceptions of health and illness. 
However, there is a growing interest in recent years in studying biomedicine as a cultural or social system and a part of the broader culture or society (Van der Geest \& Finkler 2004, Zaman 2005). Zakiya (2009) also points out that in the past few decades, more researchers including health-related professionals have moved to a more qualitative paradigm adapting and modifying these approaches to study needs of their area. In effect Boodhoo and Purmessur (2009) observed that organizational (which includes health organization) ethnography is therefore a means of looking at what people do in an organization or in other words, how people carry out different tasks at work.

It is in this regard that the research which the present article is based on was carried out on provision and use of voluntary counselling and testing (VCT) and antiretroviral therapy (ART) in two Ghanaian hospitals. The research was the first part of a larger programme that investigated the social and cultural factors that discourage or inhibit HIV-positive persons and other people from using VCT and ART services in the hospital (see Dapaah 2012, Kwansa 2013). The second component of the larger research programme looked at how family members relate to HIV-positive persons in the society and the third component studied how HIV/AIDS-related policies are formulated and implemented in Ghana.

Specifically, this article seeks to illustrate the rigorous processes/procedures social scientists need to go through to enter the hospital and the peculiar strategies they have to adopt in order to collect data in the hospital setting. Further, it tries to show how actors in the facility (which includes biomedical professionals, patients/clients and their relatives) generally perceive social scientists who conduct social science research and particularly ethnography in the hospital setting. The article thus sheds light on the experiences of an ethnographer in the hospital and how he continuously negotiated his presence to collect data in a setting which is often considered the domain of biomedical professionals.

The paper is in three parts. The first part looks at how the researcher obtained permission for the study; how he gained entry into the hospital and the strategy he employed to collect data. The second part examines the way and manner the researcher was received in the hospital. The last segment is a discussion of issues arising from the researcher's experience in the hospital, and their implications for future ethnographic studies in the area of biomedicine. Issues that were looked at in the discussion included; the lack of representation of social scientist on ethical clearance committees of Ghana Health Service and study hospitals, the perception that hospital setting is for only health professionals, the stigmatising nature of HIV and implication of doing fieldwork at home.

The article concludes by making a case for more ethnographic studies in the biomedical setting.

\subsection{Setting and methods}

The two hospitals in which the research was carried out were among the few health facilities in the Ashanti Region which benefited from the initial scale-up of VCT and ART in Ghana from 2004-2009. The region is the most heavily populated region of Ghana with 4,725,046 people according to the results of the 2010 Population and Housing Census (GSS 2010). Currently, the population of Ghana is estimated at 26,000,000 and 27,000,000 according to the Ghana Statistical Service with HIV prevalence rate of $1.67 \%$ as at the end of 2017 (NACP, 2017).

Data was compiled using qualitative research methods including participant observation in the diverse clinical settings where health workers and patients interact, informal conversations and in-depth interviews. These were complemented with a review of hospital records and socio-demographic characteristics of clients. During field research, the author participated in daily activities in the VCT centres and ART clinics and observed interactions between health workers and clients, asking questions when necessary for clarification. With the consent of patients and health workers, the researcher also participated in counselling sessions and observed interactions between counsellors and clients. More than forty health workers in the VCT centres and ART clinics were observed. He engaged in conversations with health workers and clients to gain insight into his observations about provision and use of services in the centres and clinics. Unlike interviews, which are often formal, the researcher did not guide informal conversations with health workers and clients to reduce desirability bias. In-depth interviews were conducted with health workers and clients on service provision and use. In all, 24 health workers who provided counselling, testing and treatment were selected and interviewed, including six nurses, five medical doctors, one pharmacist, one pharmacist technologist, two laboratory technicians, one disease control officer, five counsellors, one health assistant and two cleaners. Twenty-two (22) clients were also interviewed including twelve women and ten men. 
Interviews were recorded and transcribed by the researchers. With regard to data collected through participant observation and informal conversation, there searcher made a mental note of issues and actions that might be of interest to the study. I then intermittently withdraw to one of the staff rooms to write down what I had noted and later expand on them while at home. The transcribed interviews and the expanded notes from participation, observation and conversation were carefully read through and broken down into meaningful units and manually coded. The codes were grouped in broad categories that were defined and constructed on the basis of the data collected in line with Green and Thorogood's [14] ideas on coding and analysis of qualitative data. A summary was made for each category building on the common and recurrent responses as well as on different viewpoints. Various themes were identified based on the categories and illustrative quotations were also selected for presentation and discussion.

Ethical clearance was granted from the Ethics Committees of Ghana Health Service and the study hospitals. Consent was received from health workers and clients of the VCT centres and ART clinics. Research was carried out over fifteen months divided into two periods: the first phase of fieldwork was from August 2007 to July 2008 and the second phase of fieldwork (follow-up fieldwork) was between November 2009 and January 2010. Study participants were assured that whatever information they gave would be kept confidentially. They were also assured of anonymity in publication.

\section{Findings}

\subsection{Gaining access}

The 'field' for this study was the hospital. Studies have shown that it is often difficult for social scientists to gain entrée into medical settings for various reasons. For instance, the resistance of physicians to external review or regulation of professional prerogatives (Freidson 1970), and non-public spaces or 'backrooms' in medicine such as operating theatres which are supposed to be entered by only physicians and nurses (Pope 2005) can limit social science research. Van der Geest (1989) discussed the case of social science research that took place in a Dutch cancer hospital and resulted in conflict when the data were published, to the extent that the report was banned and destroyed by court order. In their introduction to a special issue on hospital ethnography, Van der Geest and Finkler (2004) suggest that the defensiveness of hospital authorities and their hesitation to allow observers in their workplace might be one possible explanation for the limited number of ethnographic studies being carried out in hospital settings, particularly in Western countries.

However, in the case of the present study, the researcher did not encounter serious difficulties in gaining access possibly due to the access route chosen. I used gatekeepers in the hospitals and to some extent my identity as a native of Ghana to gain [access to the field (see also Zaman 2003, Gerrits 2010). Gatekeepers are key people or officials in the hospitals who often influence decision-making. I used such people as intermediaries in two different ways: (i) to get permission or approval for the research. (ii) To gain entry into hospital setting for data collection.

\subsubsection{Permission}

The research team of the larger project which this paper is based on had to obtain two separate ethical clearances for data to be collected in the study hospitals. To get permission from the Ghana Health Service for the study was tortuous to say the least. The process of obtaining permission for the study began with the submission of an application to the ethics committee of Ghana Health Service which is the implementing agency of all healthrelated policies of the Ministry of Health, Ghana. Though the first phase of data collection had been scheduled for twelve months however, it took the ethics committee seven months to give clearance to the study which implied that the researcher was left with barely five months to collect data for the first phase of the research. The ethical clearance process was characterised by many queries back and forth, some of which were petty and unnecessary to some extent. For instance, the committee insisted that the larger research programme as mentioned earlier had to state the exact number of study participants. The researchers responded that in most qualitative research, the number of participants is often not clearly stated in the planning stages since it is the fieldwork situation that determines this. The committee initially objected to the researchers' explanation, which delayed approval for the study for another two months.

When it became obvious that the ethical clearance by the Ghana Health Service would take longer than expected and possibly delay the start of data collection, I got the support of a hospital administrator in one of the study hospitals to start some data collection activities while I waited for approval. 
The administrator took my request to the hospital management and I was given internal permission to carry out some limited data collection activities such as interacting and conversing with care providers and clients in the VCT centre and ART clinic.

In the case of the other study hospital, it is autonomous from Ghana Health Service and has its own committee that often gives approval for research to be carried out in the hospital. The gatekeeper I used to gain access in this facility was the director of the medicine department of the hospital. After submitting an application for ethical clearance for the study in this hospital, the researcher contacted him first to get his personal approval for the study. The Director made a case for the application to be approved at the ethics committee. It took the hospital's ethics committee a little over two months to give the study the go ahead.

\subsubsection{Entry into hospital setting}

After the study was given approval by the ethics committee of the hospital, the Director of Medicine who acted as the gatekeeper introduced me to the nursing officer-in-charge of the ART clinic and asked her to giveme the necessary support to carry out the study. This set the stage for me to start fieldwork activities in the ART clinic and the VCT centre without problems.

Similarly, in the other Hospital, the hospital administrator who had acted as gatekeeper also helped me gain entry to the hospital setting, in this case by introducing me to the medical doctor in charge of the HIV/AIDS programme in the hospital. The administrator then asked the medical doctor to support me so that I could carry out the study in the VCT centre and ART clinic. On the first day in the hospitals to start data collection activities, the nursing officer and the medical doctor respectively introduced me to staff in the clinics and the voluntary counselling and testing centres as a researcher/student. Thus, with the help of the two gatekeepers I easily gained access to the hospitals for data collection.

\subsection{Taking on a role}

Once I had gained access to the hospital setting, I still needed to take on a role in the centres and clinics in order to collect data. According to Van der Geest and Finkler (2004), the canons of anthropological research pose special problems for carrying out fieldwork in a hospital, especially how to realize the participatory aspect of the research. To overcome this challenge so that the researcher could be a 'natural' person with a continuous presence in the hospital to collect data, they have suggested three possibilities to choose from: joining the staff, the patients or the visitors. However, in the specific case of this study, I took on the role of an academic researcher as opposed to any one of the three possibilities suggested by Van der Geest and Finkler (2004).Through this role, I collected data with some reasonable level of neutrality among staff, clients and visitors. I clearly introduced myself to health workers, clients and visitors as a researcher who was interested in knowing more about the provision and use of VCT and ART from the perspectives of health workers and clients. As a researcher, I was in the centres and clinics for a period of fifteen months as indicated earlier to collect data for academic purposes. In addition, I explained that the data I was collecting would be used to improve the quality of services provided as part of the national campaign to reduce the spread of the HIV/ AIDS disease in Ghana.

The decision not to take any of the roles suggested by Van der Geest and Finkler (2004) was largely due to the belief that there was no cause to suspect that the study would not be given approval by the ethics committees of the Ghana Health Service or the hospitals involved in the research. Green and Thorogood (2005) point out that the need for researchers to be pseudo-patients for instance to collect data in the hospital arises when there is the likelihood that ethics committees would not approve the study. I was accordingly introduced to health workers and clients as a student/researcher (as mentioned earlier) who wanted to know more about the services they provide. Nevertheless, I sometimes played the role of a health worker in certain situational demands in the facilities which made some health workers and clients as well as some relatives of clients who accompanied them to access services to interpret my presence in the HIV voluntary counselling and testing centres and clinics in various ways as explained in below:

\subsection{How the researcher was received in the hospital setting}

\subsubsection{Health Workers}

In relation to health workers, I was often confronted with the following questions: "Who are you? Are you a medical doctor, a trained nurse, a student nurse or a counsellor? In which hospital were you working before you came here?" 
These questions imply that some health workers were more interested in knowing my professional background as a health worker and not as an academic researcher. The questions were probably based on the belief that the health care setting is the preserve of health professionals, so anyone seen working in the hospital must be a doctor, a nurse or a pharmacist.

I was also suspected of being a monitoring and evaluation officer who was in the facilities to assess their work and write a performance report for the hospital authorities. It follows that some health workers in one of the counselling and testing centres objected to the study being carried out in the facility. During interactions with the staff to explain more about why I needed to do the study in the centre, a counsellor commented as follows:

We, staff are not certain that you [researcher] are not here to judge our work because you are not a trained counsellor...

Another counsellor remarked that:

... If you [researcher] want to know how VCT services are provided, you can do that without sitting in counselling sessions to see how we do our work...You are even not a trained counsellor, so how can you know that we are doing our work well or not...?

Some health workers also took me for a national service person or an Intern on attachment for on-the-job-training in the centres and clinics. In Ghana, it is obligatory for students who have completed tertiary education to do one year of national service in any sector and part of the country. National service persons are often posted to the health sector to complement the work of health care professionals. There were many instances when some health workers, particularly those who worked shifts in the clinics, asked me:

My brother [researcher], you are still doing your national service here in the hospital? So, when is your service ending... or you have now been employed here permanently...?

These questions were possibly due to my long presence in the facilities, which lasted almost the same as the length of time as it does for one to do his national service.

\subsubsection{Clients}

Many clients received the researcher as a new health worker in the centres or clinics. Some clients addressed me as 'Doctor' but this is not new in Ghanaian hospitals. It is common for clients and visitors to the hospitals to address particularly male health workers as Doctor (Dòkita). They also addressed a female health worker as 'Aunt' or 'Madam Nurse' (Auntie orMaame Nurse). This distinction between male and female health workers by clients and visitors most of whom are illiterates is perhaps based on the assumption that it is only male health workers who are called doctors while their female counterparts are called nurses. In that case some clients and visitors do not even know that there are male nurses. This way of receiving the researcher may be due to the health worker role I sometimes played in the centres and clinics. During fieldwork, I in some cases helped health workers to give out laboratory test results and treatment folders to clients before they were attended to by the nurses and medical doctors.

Other clients also approached me with some technical health problems and questions about their treatment because they thought that as a health worker, I could solve such problems or answer their questions. For example, I was asked by some clients to explain why they were given a particular combination of ARV drugs different from what their colleagues were given. I often politely explained to them that I was not a professional health worker but a researcher and was not in a position to give the appropriate answer. In such cases, I always urged clients to either talk to the medical doctor who wrote the prescription or to talk to a nurse or a pharmacist who would be in better position than I to answer. It was however observed that most of the time clients were reluctant to talk to medical doctors, some of whom they described as "not being friendly" or "too formal" (wònnte wòn anim). According to clients, this makes it difficult for them to approach most doctors in an informal way with such questions about treatment and other related issues. However, one doctor in one of the study hospitals was often praised by clients as being kind and willing to help solve their problems whenever they approached him.

I was also received in the hospital setting as an 'unwelcome' friend by some clients who came from my hometown or the area near my hometown. These clients had not expected to see someone from their region in one of the clinics where this study was carried out. The body language of some of these clients from my hometown area whenever I saw them in the clinic gave the impression that they were not happy to see me. One managed to avoid any contact with me on several occasions in the clinic. 
Another client had no choice but to come to me for her CD4 count test result (the test that is taken to determine the level of a client's immune system) that was on the table where I was seated. When the client came to the table, I casually engaged her in conversation and assured her that she should not be worried that I had seen her in the clinic because I would not mention her status to other friends.

Another client initially tried to avoid contact with me by looking the other way; he did not seem to realize that I had seen him as soon as he had entered the clinic. He also came to the place I was seated to see a nurse to help him get re-supply of ARV drugs without wasting too much time in the clinic. After exchanging greetings, he asked whether I was working in the clinic. I briefly explained the research to him and added that I would be in the clinic for a period of eight months because I had already spent four months of the first phase of fieldwork. When the client heard this, he paused momentarily and shifted his attention to the nurse. The way he suddenly ended our conversation created the impression that he was uncomfortable with something. I asked to see him after he finished accessing treatment. In a conversation, I allayed whatever fears he may have had by assuring him I would not disclose his status to friends back in our hometown. I urged him not to let my temporary presence in the clinic be a barrier to his accessing treatment. The smile on his face suggested that he was relieved with the assurance I gave him.

Some clients I had attended secondary school with received me as a schoolmate and not as a researcher. I had not seen some of these clients since our schooldays. Like the clients from my hometown, some of my former schoolmates appeared to feel uncomfortable that I saw them in the clinic. I recall that on a clinic day, one of these clients who was my senior in school saw me seated with some health workers in the clinic. Under normal circumstances he would have come up to say hello as we had not seen each other since we had left school about a decade ago. However, the former senior turned his back towards me while he went through his treatment procedures. He did not seem to know that I had recognised him from a distance when he entered the clinic. Moments later, I approached him and engaged him in a conversation. It was then he said that he saw that I was seated with the health workers but he was not sure whether I was the one. He looked very sick and I had to help him round the clinic to access treatment without much delay. In the case of one other schoolmate, she came to where I was seated and wanted to know what I was doing in the clinic. She was happy to see me after so many years. I told her about the study I was carrying out in the hospital and also assured her that I would not disclose her status to other schoolmates.

There was another client who saw me as her former schoolteacher rather than a researcher. I had taught in the Junior Secondary School (which is now called Junior High School) when I did national service a couple of years back. She was also happy that we met again and willingly told me how she got the disease. During the fieldwork period, I maintained close contact with her because I was the only person apart from her mother who was aware of her status. I used to advise her on how she could manage the HIV infection and live longer during my prolonged presence in the clinic. According to her, I was the only person she was able to discuss issues about the disease with because as her former teacher she trusted that I was someone she could confide in. She pointed out that even her husband was not aware of her status.

Lastly, most of the clients saw me as a buddy in the centres and clinics. Since many clients and their adherence monitors (relatives of clients who often accompany them to the clinic for their monthly ration of ARV drugs) considered me as a friend, it was not surprising that I did not have any difficulty in getting clients to participate in this study as respondents. To them, I was someone with whom they could share their personal problems related to treatment, marriage and family with. More importantly, they saw the researcher as someone who always had time for them and lent an ear to the problems they faced at home and in the facilities. It was easy to find clients to converse with and to elicit their views on the provision and use of services as part of data collection. Clients approached me on many occasions to intercede on their behalf when they had problems with health workers. To date, I have kept in touch with some of them (including my former pupil) to keep track of how they are doing on treatment or whether they have managed to carry out some of the decisions we discussed before I left the field.

\subsubsection{Relatives of clients (Adherence Monitors)}

Likewise, some adherence monitors received the researcher as a client or adherence monitor. In the centres and clinics, apart from visitors who come there to see health workers on some other matter, people are not allowed to visit clients accessing care and treatment the way they can in the admission wards. Relatives who are adherence monitors of clients often accompany clients when they come to use services so they would help them to have easy access to the facilities. 
This suggests that if a non-health worker is seen regularly, especially in the clinic, he is assumed to be a client or an adherence monitor. My continuous presence in the clinic possibly made some relatives of clients think that I was a client who has been receiving care and treatment in the clinic. Adherence monitors who wanted to know whether I was also a client often asked; "So, when did you start accessing treatment here? How long have you being accessing treatment here? Where do you always come from to get treatment here?" In the same way, some monitors also asked questions like: "Whom did you accompany here? Did you come here with your wife? How is the person you accompanied here doing on the treatment?"I often explained to the relatives or adherence monitors that I was neither a client nor an adherence monitor but I was an academic researcher who was interested in studying how HIV/AIDS-related health care services are provided and used.

\subsection{Field work at home}

It is worth mentioning that both health workers and clients received the researcher as a native who should be given the necessary support to carry out the study. Perhaps my Ghanaian identity positively influenced the respondents to willingly participate in the study and gave me the relevant information. As indicated earlier, my Ghanaian identity to some extent facilitated the initial clearance I got from the study hospitals to start some limited data collection activities while waiting for the ethical clearance from Ghana Health Service. Besides, there were many occasions I used my previous links in one of the hospitals to obtain some useful data for the study from hospital records with little or no difficulty. These courtesies which were extended to me by fellow Ghanaians before and during data collection might not be extended to a non-Ghanaian who could not express himself or herself in the local language and easily put his or her case across for assistance or support when necessary.

\subsection{Discussion}

In view of the tortuous nature of the ethical clearance process for this study, it can be assumed that there were no social scientists on the committee vetting the research proposal for approval. This suggests that only medical scientists who were not familiar with ethnography were on the ethical clearance committee. Throughout the clearance process, the committee raised queries, some of which were trivial that delayed the actual start of data collection for several months in both study hospitals. In one of such queries, besides the one mentioned earlier, the ethical committee wanted to know how scientific the purposive sampling method adopted by study to select study participants. This was a further indication that there was no social scientist on the ethical committee who could have drawn the attention of the committee members that unlike quantitative research, non-probabilistic sampling technique is in most cases used to select study participants in ethnographic or qualitative research. This is mainly due to the relatively smaller number of people that are often selected to participate in qualitative research such as the research this paper was based on as opposed to quantitative research which often has large sample size. Besides, the study had inclusion and exclusion criteria for the selection of participants which meant that the respondents were to be selected purposively.

Perhaps, a social scientist was not on the ethical committee because social science researchers are often considered 'outsiders' in the domain of biomedical research. From this researcher's experience, it is clear that the addition of social scientists to the committees that vet health research proposals for approval in Ghana and elsewhere is long overdue. Mulemi (2010) also made a similar observation in his study in the cancer ward of a Kenyan Hospital. He indicated that hospital ethnography did not have a place in the hospital's protocol for research clearance. At the very least, social scientists could sit on these committees to help assess ethnographic or qualitative research protocols, which would promote the role of ethnography in hospital research and its findings could then be used to improve the quality of care.

Social scientists are often not easily accepted to carry out research in the hospital because the perception has been that their data collection approaches do not meet biomedical ideas of scientific research. It was not surprising that health workers in the hospitals were reluctant to accept me, as an anthropologist. Initially, I was often confronted with the questions which mainly sought to find out my professional background as a health worker. Whenever I explained to health workers that I was a social scientist who was interested in knowing more about how VCT and ART services are provided and used, I observed some reservations in the facial expression of care providers.

The qualitative research approaches I used to collect data further strengthened the doubts of health workers about the study and its motives. Similar observations have been made in ethnographic studies in the hospital elsewhere. For instance, Mulemi (2010) reported that health care providers had reservations about his study in a Kenyan hospital cancer ward. 
He attributed this to the low awareness of the value of qualitative research in promoting patient care among health professionals. Albert et al. (2008) point out those biomedical scientists or health care professionals' doubts about qualitative research in the health care setting is due to the perception that its methods are devoid of any scientific foundation. This, according to the authors is due to three opinions: its results cannot be reproduced, the researcher's subjectivity interferes at all stages of the research process, and there is no effective way to control for biases. In line with the views of Albert et al. (2008), perhaps, Goodson and Vassar (2011) are right in their observation that though to some extent, ethnography has been applied in healthcare settings and in the medical education environment, yet there is a general lack of research in the setting employing this method (see also Leung, 2002).

Health professionals, however tend to overlook social and cultural aspects of disease and care-seeking which do not always lend themselves to quantitative research. Doctors and nurses tend to be reluctant to allow non-medical observers to their work for the fear that as outsiders in the health care setting they may pass judgment on what they do in hospital (see Van der Geest \& Sarkodie 1998:1374). Health professionals dislike being watched by outsiders such as ethnographers or social scientists who do not always reveal their motive in hospital research and will later put their observations on paper criticizing them (see also Van Staa 1993). As a result, most medical professionals are therefore suspicious about ethnographic or qualitative research.

However, the initial doubts of health workers in this particular study gradually changed to cooperation with the researcher as they began to appreciate the contribution the study could make towards improvement of service provision and patient care. The use of qualitative research methods like informal conversations helped to bring health workers and clients together to share their views and opinions on how services are provided. The informal nature of such conversations enabled clients in the clinics especially to freely express their perceptions on the quality of services provided. Clients also used the conversations to let out their pent-up emotions about certain practices of some health workers towards them. For instance, in a conversation which involved this researcher, two clients and a health worker, a client complained about the disclosure of her status to the grandmother by a nurse in the delivery ward. According to the client, the disclosure led to her rejection by the grandmother. The client could not have expressed her personal views in a clinical encounter for fear of being labelled as bad or uncooperative client.

Thus, the present study has shown that clients felt comfortable to discuss issues on service provision with health workers in informal conversations. This may encourage health workers to more often engage in conversations with clients outside clinical encounters and know more about their problems or challenges on care and treatment. Moreover, the personal views of clients in this study gave strong evidence about areas of client care and treatment that need improvement.

Further, this study has shown that ethnography in the hospital does not end up criticizing or antagonizing biomedicine per se. It has provided information about clients' individual level of health care needs beyond figures that health workers often use as indicators to improve upon quality of services to satisfy clients. It is worth mentioning that this study has established that health workers mostly behaved well towards people living with HIV/AIDS in their interactions contrary to the often held view in earlier studies that health workers tend not to treat them well (see Dapaah, 2012 and Dapaah, 2016). This finding recognises the efforts of health workers to provide quality care and treatment to the satisfaction of clients. Similarly, Goodson and Vassar (2011) pointed out those ethnographic studies in health care settings help to get to the root issues about patient care rather than tracking behaviour which is often akin to quantitative research. From this perspective, Goodson and Vassar (2011) further contend that ethnographic studies in healthcare settings lead to finding real solutions to provision and use of health care services rather a trial and error process.

It is obvious from the discussion so far that stigma is the underlying reason for the varied ways the researcher was received in the facilities by both health workers and clients and their relatives (Adherence monitors). According to Niehaus (2007), the main cause of stigma is the association of the disease with death rather than sexual promiscuity. Writing about South Africa, he acknowledges that this assertion is not entirely new but it shifts the emphasis for the cause of HIV-related stigma from immoral sexual behaviour or unprotected sex to fear of death. He observes that although immoral sexual behaviour is frowned upon by society, the sentiments against it are not strong enough to lead to the persistent stigma related to the disease and positive persons. In some African cultures, men are allowed to marry more than one woman and teenage pregnancies due to pre-marital sex are also common. 
The association of the disease with death and the subsequent stigma has also been reported in other studies. For instance, studies by Ashforth (2002:116) and Viljoen (2005) report that respondents in South Africa described AIDS as 'a waiting room for death' and positive persons as 'dead before dying.' According to Niehaus, this perception of HIV infection illuminates many aspects of people's responses to the disease, including the (non) use of health care services. It also helps to explain why most clients preferred to use services in privacy and hide their status from relatives and others

These views about the disease are common in Ghana. In a study on the disease among the Akan in Ghana, Crentsil (2007) observes that positive persons are often stigmatised because the disease is believed to be incurable and the fear that the infected person is likely to die of it. Likewise, the pain and suffering the person goes through before HIV-related death is generally considered as shameful and disgraceful, and in some cases leads to perfunctory funerary rites. This is often due to the fear of contagion associated with the corpse of person who died of HIV infection. Such death is described as 'bad death.' The concerns of clients in this study on the social risks associated with the disease resonate Niehaus and Crentsil's findings that HIV is a deadly disease. The fear of death associated with the disease and the shame that often characterises HIV-related death could frighten relatives and others to distance themselves from its sufferers in the form of stigma.

It is against this background I interacted and communicated with both health workers and clients to collect data in the health facilities. Though none of the health workers asked about my HIV status, it could be possible that some of them thought I am HIV-positive person and disguised myself as a researcher to collect data and avoid stigmatisation. As Van der Geest and Finkler (2004) points out there have been many instances in ethnographic research in which researchers adopted the role of a staff, visitor or patient to hide their true identity to collect data in the biomedical setting. An example was the pseudo patient role Van der Geest and Sarkodie (1998)used to collect data in a Ghanaian hospital. In this regard, it could be that health worker, clients and adherence monitors asked me those numerous questions ostensibly to glean some information from me to either confirm their suspicion of the researcher as HIV patient or otherwise.

With regards to clients who came from my home town area including; those I attended Secondary School (now Senior High School) were obviously not comfortable with my presence in the hospitals. This was mainly due to the fear that I would disclose their status to other friends and acquaintances for them to be stigmatised. The persistent stigma associated with HIV/AIDS in Ghana as observed earlier, has compelled many patients suffering from the disease to access care and treatment outside of their hometown area or at facilities far away sometimes in another region. This, the patients often believed would help them access services in privacy and hide their identity as HIV-positive persons because the possibility of meeting people who might know them in such hospitals is very low (see Dapaah \& Senah, 2016 and Dapaah \& Rachel, 2016). In other words, the uptake of services outside one's area of origin is a coping mechanism against stigmatization or a 'safety net'; to avoid identification as HIVpositive persons by relatives and neighbours. This was exactly the reason why clients from my hometown area decided to use services in another region only to find me in those facilities. It can thus be concluded that the discomfort some of the clients had with my presence in the centres and the clinics explains the different ways they received me in the facilities.

Lastly, my identity as a Ghanaian might have also influenced how I was received in the facilities by both health workers and clients. Throughout my fifteen months stay in the facilities, I was seen first and foremost as a native or Ghanaian and secondly as an academic researcher. However, a major challenge I anticipated in this research was my background as a Ghanaian studying at home and its implications for the collection of objective data during fieldwork. There is the general perception that when doing fieldwork in one's own culture or country, a researcher runs the risk of developing 'over rapport' with the research subjects which might hamper the attainment of objective information (Zaman 2005:31). Unlike the anthropologist in a small-scale society who lives and works twenty-four hours a day with an unchanging population, the encounter I had with the health workers in this study was confined to working hours and the client population was always changing. Van Dongen (1998) also argues that being an anthropologist at home does not mean that the identities of the anthropologist and people in the field are similar. On the contrary, in this study, the participants saw me as one of their own which to a large extent might have influenced how they received me in the centres and clinics.

Consequently, my background as a Ghanaian studying in my home country was an advantage in many respects. For instance, as a native of Ghana, my presence in the centres and clinics did not indicate anything unusual, unlike in the case of a 'white' or 'coloured' anthropologist in a 'black' population. 
Moreover, during most part of the day, the centers and clinics were public places where relatives came with the sick, and food and newspaper vendors and others came to do business. In effect, I was taken as a relative who was accompanying a client or someone who was in the clinic to do business with health workers. With regard to language, I speak the same Akan language (Asante Twi) as most of the health workers and clients, so when they told their stories, I easily understood the meaning of the important events and even the layered meanings of their body language. This is not the same for anthropologists who study other cultures and have to go through a fairly long and painful period of adjustment to accumulate knowledge, competence and sensitivity. Being an Akan and therefore an 'insider' greatly reduced the cognitive and emotional efforts necessary to adjust and understand an otherwise foreign culture.

Notwithstanding the relative success of doing fieldwork in my homed country, I was always mindful of the need to 'maintain the balance between intimacy and distance' as posited by Wind (2008:86). In her ethnographic study in a highly specialized hospital in Demark where she comes from, Wind (2008) pointed out that she had to convince other anthropologists including her that it is possible for a native anthropologist to engage in ethnography in his/her home country. Thus, as a health professional herself, she did not go into the hospital setting to carry out the study as a nurse and neither as a Dane but rather as an ethnographer in order for her to be 'neutral' and collect data. Likewise, as a Ghanaian ethnographer in Ghanaian hospitals, though I was not a health professional, I had to also maintain the balance between my identity as a Ghanaian and an academic researcher and not to be carried away by my familiarity with some health workers and clients in order for me to be neutral and collect objective data as an ethnographer and not as a Ghanaian per se.

In conclusion, the empirical data and discussion above show that the anthropological method is not common in the biomedical setting and this might have influenced the various ways the researcher was received by particularly health workers and clients. There is therefore the need for more hospital ethnography to make it popular in the medical settings and among health workers. Such studies would also demonstrate the important contribution ethnographic research can make to quality health care delivery. More importantly, the paper seeks to further strengthen the position of social scientists and for that matter anthropologists that biomedicine and the environment within which biomedicine is practised are equally 'fields' that are worth researching on like other social institutions.

\section{Competing interests}

The author declares that he has no competing interests

\section{Author contribution}

JMD was the researcher and the sole author of the manuscript.

\section{Author details}

Department of Sociology and Social Work, Faculty of Social Sciences, Kwame Nkrumah University of science and Technology, Kumasi, Ghana

\section{Acknowledgements}

I would like to express my gratitude to the Netherlands Organization for Scientific Research, NOW-WOTRO which funded the entire $\mathrm{PhD}$. research programme which the current article is based on and also cited in the list of references. I thank all the health workers and clients of the VCT centres and ART clinics of the hospitals which participated in the study for the patients with which they answered the many questions I asked. I further acknowledge the contribution of my two other colleagues - Dr. Rachel Spronk (a post-doctoral student) and Benjamin K. Kwansa (a PhD. Candidate) on the research programme towards the completion of this paper.

\section{References}

Al-Busaidi, O. Z. (2008). Qualitative research and its uses in health care. Sultan Qaboos University Journal, Vol 8. Iss. 1. pp 11-19.

Ashforth, A. (2002). An epidemic of witchcraft? Implications of AIDS for the post-Apartheid state. AfricanStudies, 61:116.

Boodhoo, R \& R. D. Purmessur (2009). Justification for qualitative research in organizations: A step forward. The Journal of Online Education. New York. 
Crentsil, P. (2007). Death, ancestors, and HIV/AIDS among the Akan of Ghana. Ph.D.dissertation, University of Helsinki.

Wind, G, (2008). Negotiated interactive observation: Doing fieldwork in hospital settings. Anthropology and Medicine, Vol. 15, No. 2, 79-89

Dapaah, M. J. \& K. A. Senah (2016). HIV/AIDS clients, privacy and confidentiality: The case of two health facilities in the Ashanti Region of Ghana. BMC Public Health Journal, 17 - 41.

Dapaah, J. M. \& R. Spronk (2016). When the clinic becomes a home. Successful VCT and ART services in a stressful environment. SAHARA-J: Journal of Social Aspects of HIV/AIDS. Vol. 0 No.0, 1 - 10.

Kwansa, B. K. (2013). Safety in the Midst of Stigma. Experiencing HIV/AIDS inTwo Ghanaian Communities, Leiden, African Studies Centre.

Dapaah, J. M. (2012). HIV/AIDS treatment in two Ghanaian Hospitals: Experiences of patients, nurses and doctors,Leiden: African Studies Centre, 2012.

Holloway, I. (1997). Basic concepts for qualitative research research. Oxford: Blackwell Science.

Leung, W. C. (2002.). Why is evidence from ethnographic and discourse research

needed in medical education: the case of problem-based learning. Med. Teach. 24: 169-172.

Goodson, L. \& M. Vassar (2011). An overview of ethnography in healthcare and medical education research. $J$. Educ. Eval. for Health Prof. 8: 4: 1-7.

Freidson, E. E. (1970).Professional dominance: The social structure of medical care. Chicago: Aldine.

Gerrits, T. (2010) Dynamics of patient-centred practices in a Dutch fertility clinic. Ph.D. dissertation, Amsterdam School for Social Science Research, University of Amsterdam.

Ghana Statistical Service (2010). Ghana national population census report. Accra, Ghana.

Green, J. \& N. Thurgood (2005). Qualitative methods for health research. London: Sage Publications.

Kwansa, B.K. (2013). Safety in the midst of stigma. Experiencing HIV/AIDS in two Ghanaian communities. PhD Dissertation, University of Amsterdam (draft).

Mulemi, A. B. (2010).Coping with cancer and adversity: Hospital ethnography in Kenya. Ph. D. dissertation, University of Amsterdam/Leiden: African Studies Centre.

NACP/GHS (2016).2016 HIV Sentinel Survey Report, Accra, Ghana.

Niehaus, I. (2007). Death before dying: Understanding AIDS stigma in the South African Lowveld. Journal of Southern African Studies 33(4):845-860.

Patton, M. Q (2002). Qualitative evaluation mwthods. CA: Sage Publications.

Pope, C. (2005). Conducting ethnography in medical settings. Medical Education 39: 1180-1187.

Van der Geest, S. (1989). Censorship and medical sociology in The Netherlands. Social Science and Medicine 28: 1339-1342.

Van der Geest, S. \& K. Finkler (2004). Hospital ethnography: Introduction. Social Science and Medicine 59: 1995-2001.

Van der GEEST, S. \& S. Sarkodie (1998). The fake patient: A research experiment in a Ghanaian hospital. Social Science and Medicine 47: 1373-1381.

Van Dongen, E. (1998). Strangers on terra cognita. Authors of the other in a mental hospital. Anthropology and Medicine 5(3): 279-293.

Van Staa, A. (1993.) Myth and Metronidazole in Manila. The popularity of drugs among prescribers and dispensers in the treatment of diarrhoea. Master Thesis, University of Amsterdam.

Viljoen, F. (2005). Disclosing in the age of AIDS: Confidentiality and community in Conflict. In: F Viljoen et. al (Eds.) Righting stigma: Exploring a rights-based approach to addressing stigma, pp. 68-87. Human Rights Research Unit, University of Pretoria.

Zaman, S. (2005). Broken limbs, broken lives: Ethnography of a hospital ward in Bangladesh. Amsterdam: Aksant. 